Revista de Psicología Vol. 29 (2), 2011 (ISSN 0254-9247)

\title{
Resiliencia en el maltrato infantil: aportes para la comprensión de factores desde un modelo ecológico ${ }^{1}$
}

\author{
Gabriela Morelato ${ }^{2}$ \\ INCIHUSA / CONICET, Argentina
}

\begin{abstract}
El presente trabajo integra conceptos vinculados a la resiliencia en el ámbito del maltrato infantil desde el modelo ecológico, haciendo énfasis en la descripción de factores de riesgo y protectores, a fin de acercarnos a la comprensión de sus modalidades de interacción como proceso dinámico. Esto implica enfocarse, no solo en las graves consecuencias que el maltrato produce, sino también en el estudio de los recursos infantiles que permiten a los niños continuar con su desarrollo a pesar del riesgo. Se considera que la comprensión de la resiliencia en el ámbito del maltrato infantil desde este modelo interaccional, bidireccional y recíproco puede acercarnos a pensar mejores modos de promover potencialidades en la infancia.

Palabras clave: resiliencia, maltrato infantil, modelo ecológico.
\end{abstract}

\section{Resilience in child maltreatment: Contributions to understanding the significant factors in the process from an ecological model}

The present study aims to integrate resilience concepts within the context of a theoretical model for child maltreatment, the ecological model, as well as to describe risks and protective factors leading to a better interaction as a dynamic process. Thinking deeply about maltreatment from the point of view of resilience implies focusing on the serious consequences it produces, as well as on the assessment of children's resources to continue growing and developing despite the risks. We may promote children's potential by understanding resilience within a child maltreatment framework based on this model (interactional, bidirectional and reciprocal).

Keywords: Resilience, child maltreatment, ecological model.

1 Estudio realizado gracias a la beca de postgrado Tipo I otorgada por CONICET, Argentina, para la tesis doctoral "Evaluación de la resiliencia en nińos víctimas de maltrato familiar" dirigida por las Dras. Mirta Ison y Mabel Labiano.

2 Doctora en Psicología. Becaria Posdoctoral del CONICET, Docente de la Universidad del Aconcagua, Argentina. Contacto: Instituto de Ciencias Humanas, Sociales y Ambientales INCIHUSA-CONICET-CCT, Av. Adrián Ruiz Leal s/n, Parque Gral. San Martín, Cdad. C. Correo 131 M5502 IRA Mendoza, Argentina; gmorelato@mendoza-conicet.gob.ar 

El maltrato infantil es un problema social grave que, como fenómeno complejo, es atravesado por diversas áreas que involucran aspectos sociales, culturales, históricos, económicos y de salud, tanto de naturaleza física como psicológica. Es indudable que sus consecuencias afectan enormemente el desarrollo del niño, el adolescente y el adulto, quienes evidencian dificultades en casi todas las dimensiones evolutivas (Cichetti \& Lynch, 1993; Cichetti \& Rogosch, 1997; Lessinger Borges, Haag Kristensen \& Dalbosco Dell'Aglio, 2006). No obstante, en la experiencia de atención y seguimiento de casos vinculados a esta problemática es posible observar distinciones en el modo en que cada uno de los niños y niñas puede enfrentar las diversas situaciones de riesgo en sus distintos niveles de gravedad y cronicidad. Un gran número de nińas y nińos manifiestan trastornos severos una vez que se los preserva del peligro inminente. Sin embargo, otras y otros desarrollan resiliencia, mostrando buena adaptación a los diferentes contextos interpersonales, así como afrontando con éxito y con buenas perspectivas de evolución las situaciones estresantes (Cicchetti, 2001; Cicchetti, Rogosch, Lynch \& Holt, 1993; Morelato, 2009).

En el último tiempo, la resiliencia ha sido investigada desde diferentes ámbitos. En América Latina su estudio se hace cada vez más frecuente dadas las circunstancias sociales (Melillo \& Suárez Ojeda, 2001; Melilllo, Suárez Ojeda \& Rodríguez, 2004). Sin embargo, más allá de las generalidades en el área del maltrato infantil, se considera útil analizar los principales factores que forman parte de este entorno de riesgo específico. A partir de lo expresado, el objetivo de este trabajo es integrar conceptos vinculados a la resiliencia en el ámbito del maltrato infantil desde el modelo ecológico (Belsky, 1980, 1993; Bronfenbrenner, 1979, 1987), haciendo énfasis en la descripción de factores de riesgo y factores protectores, a fin de acercarnos a la comprensión de sus modalidades de interacción como un proceso dinámico. 


\section{Antecedentes teóricos}

La resiliencia es un término tomado de la física para explicar la propiedad de algunos cuerpos de recobrar su forma original luego de haber sido sometidos a altas presiones. Sin embargo, su aplicación en las ciencias humanas presenta una connotación diferente. Surge a partir de la necesidad de explicar cómo numerosos individuos, inmersos en situaciones adversas de alta tensión, podían efectuar un proceso diferente a lo que era esperable: podían responder de una manera positiva y adaptada, pese a vivir y crecer en condiciones riesgosas (Kotliarenco, Cáceres \& Fontecilla, 1997).

Aunque existen diversas definiciones sobre el concepto resiliencia, puede decirse que es fundamentalmente un proceso que permite retomar algún tipo de desarrollo a pesar de la ocurrencia de una situación traumática (Cyrulnik, 2003b). En esta línea, se la ha definido como un proceso dinámico, que depende de factores internos (personales) y externos (contextuales), tanto de riesgo como protectores. Se entiende que tanto el conjunto de factores de riesgo como el conjunto de factores de protección conforman dimensiones. Las dimensiones de riesgo y de protección interactúan entre sí para generar un mecanismo que hace posible darle continuidad al desarrollo o a algunos aspectos del mismo, a pesar de las circunstancias (Morelato, 2009).

En base a este planteamiento, es importante destacar que la resiliencia alude a la presencia de una modalidad particular de riesgo y a una respuesta del individuo o grupo mucho más favorable de lo que se esperaba para la trayectoria del mismo. Como fenómeno general, la resiliencia se vincula a una serie de factores que interactúan entre sí a modo de mecanismos dinámicos: los factores de riesgo y los factores protectores. Es de destacar que los factores de riesgo hacen referencia a condiciones cuya presencia facilita la aparición de resultados negativos e indeseables para el desenvolvimiento humano tales como problemas físicos, psicológicos y sociales (Casol \& De Antoni, 2006). En contraste, de acuerdo con Rutter (1990, 1995), los factores protectores son influencias que modifican, mejoran o alteran la respuesta de una persona a algún peligro que predispone a un resultado no adaptativo. 
Estas pueden ser circunstancias, atributos, personas o sucesos que inciden positivamente en los resultados del desarrollo, producto de su interacción con el riesgo, lo cual las transforma en factores de resiliencia. De este modo, la presencia de riesgo puede desembocar en una doble vía: puede llevar a la vulnerabilidad, es decir a la presencia de respuestas negativas en el individuo tales como disminución de autoestima, trastornos de personalidad y depresión, entre otros, o puede llevar a la resiliencia, es decir a que la persona pueda darle continuidad a su desarrollo o a una gran parte de él, a pesar del riesgo. La inclinación hacia una u otra vía dependerá del impacto que tengan estos factores en el desenvolvimiento del individuo (Casol \& De Antoni, 2006).

Es preciso señalar que hablar de resiliencia no implica invulnerabilidad en el sentido que el nińo, adolescente o adulto sea inmune al riesgo. Al contrario, se debe subrayar, tal como lo plantea Cyrulnik (2003b), que la experiencia traumática o adversa deja huellas en la vida del individuo y por ello solo se puede hablar de resiliencia cuando se ha producido un trauma que, aunque se instala en la historia del sujeto y no se olvida, permite la recuperación de algún tipo de desarrollo. Esta continuidad dependerá de los recursos internos y externos con los cuales cuente el niño.

En esta línea se ha incluido el concepto de dimensión (ver figura 1). Las dimensiones son entendidas como áreas que agrupan factores. El conjunto de factores que conllevan a una mayor predisposición a la vulnerabilidad se denomina dimensión de riesgo. El conjunto de factores que conducen a mayor probabilidad de generar un mecanismo de protección forman parte de la dimensión de protección. El producto de la interacción de ambas dimensiones genera una combinación particular que puede dar lugar a un mecanismo de protección o, en su defecto, a un mecanismo de vulnerabilidad. En el primer caso hablamos de la existencia de un proceso de resiliencia que se expresa en los resultados de la competencia y en la adaptación positiva. Cuando la disposición y combinación de los factores generan un mecanismo de vulnerabilidad es esperable encontrar retrasos en el desarrollo y sintomatología grave, entre otros factores desfavorables (Morelato, 2005, 2009). 


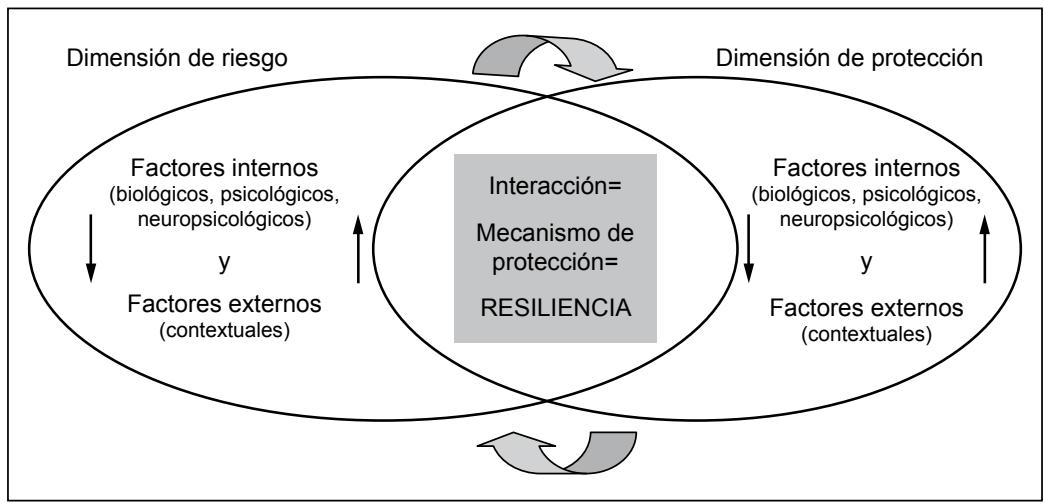

Figura 1. Interacción entre dimensiones de riesgo y de protección en el proceso de resiliencia (Morelato, 2005, 2009).

En la figura 1 se observa la presencia de factores externos e internos que pertenecen tanto a la dimensión de riesgo como a la dimensión de protección, la interacción entre factores y entre las dimensiones. Se debe explicar que los factores de riesgo y de protección no son fijos. Por ejemplo, la familia que en una situación de guerra es un agente de apoyo y protección, constituye un factor de riesgo en las situaciones de maltrato infantil. Sin embargo, cuando nos adentramos en contextos específicos, los factores de riesgo y los factores protectores pueden ser más definidos. En otras palabras, los factores que conforman las dimensiones de riesgo y de protección no son generales pero se tornan más significativos según la circunstancia. Por ejemplo, en el maltrato infantil el cuidador violento siempre es el principal factor de riesgo. Es así que según se combinen e interactúen en un proceso, pueden acercarse a la vulnerabilidad o a la resiliencia. Esta combinación puede comprenderse de diferente modo: a veces el riesgo puede ser contrarrestado por el apoyo a modo de modelo compensatorio. Por el contrario, si el riesgo no es excesivo, puede generar competencias desde un modelo de desafío. Finalmente, también puede evidenciarse un modelo de adaptación en el cual los factores que atenúan el efecto del riesgo producen adaptación adecuada (Werner, 1993). 


\section{El modelo ecológico}

Luthar, Cichetti y Becker (2000) reconocen la importancia de distinguir los múltiples contextos que afectan el desarrollo infantil, especialmente tres: la comunidad (vecinos y soportes sociales), la familia y el propio niño. En esta línea, la teoría bioecológica (Bronfenbrenner, 1979, 1987) contempla el desenvolvimiento humano en forma ampliada, focalizado en las interacciones mutuas entre el individuo y su medio ambiente. Esta propuesta es útil para comprender la influencia dinámica de múltiples factores en el desarrollo del niño o adolescente. Para este modelo, el desarrollo humano es un proceso dinámico, bidireccional y recíproco donde el niño reestructura de modo activo su ambiente y recibe el influjo de los factores vinculados con él. El modelo está compuesto por cuatro núcleos que están interrelacionados y se denominan: persona, proceso, contexto y tiempo. El primer núcleo es la persona, vista con sus características innatas, biológicas, emocionales y con aquellas constituidas por el contacto con el ambiente. El proceso o segundo núcleo es la forma en que la persona interactúa con el contexto, sus características particulares, la forma cómo vive sus experiencias y el rol que desempeña en su vida (Bronfenbrenner como se citó en Casoll \& De Antoni, 2006). Los procesos o modos de interactuar entre personas, objetos, símbolos o contextos pueden ser distales o proximales. Se denomina distal y proximal a los extremos de un continuo en el que algunas variables son propiamente más lejanas que otras. Así, existiría una cadena causal que comienza con la variable distal (e. g. pobreza), actuando a través de sus consecuencias sobre las variables mediadoras (e. g. ansiedad maternal), para afectar al niño a través de una o más variables proximales (e. g. irritabilidad de la madre). Según el modelo de Bronfenbrenner (1979), los factores distales serían los macrosociales, ya que no afectarían directamente al nińo pero tendrían un efecto sobre algunos de los procesos o comportamientos que ocurren a nivel proximal (Baldwin, Baldwin \& Cole, 1992). Estos autores explican que un proceso de intervención puede ser más viable cuando tiene como objetivo la modificación o el refuerzo de algunas variables proximales. No ocurre lo mismo con los factores distales, quedando estos 
más bien en manos de las decisiones políticas de tipo macrosocial, no sujetos a intervenciones psicosociales específicas.

En el modelo bioecológico de Bronfenbrenner (1979), el tercer núcleo es el contexto, compuesto por un conjunto de cuatro sistemas concéntricos con interconexiones, agrupados, interdependientes y dinámicos. Son niveles que ocurren simultáneamente y que van desde el contacto más íntimo del niño con los responsables de sus cuidados, hasta los contextos sociales más amplios. Tales sistemas son denominados microsistema, mesosistema, exosistema y macrosistema. El microsistema está relacionado con las actividades y roles del niño en su medio inmediato. Se refiere al conjunto de actividades y relaciones interpersonales vivenciadas en el entorno específico y a través del contacto directo, correspondiendo a la más íntima interacción entre personas y ambiente, basadas en reciprocidades y estabilidad. Señala los aspectos cotidianos de la casa, la escuela, el trabajo y las relaciones directas con los padres, hermanos, colegas y profesores.

El entrecruzamiento de varios microsistemas que envuelven a una persona conforma el mesosistema. Este sistema incluye vínculos entre familia y escuela, o familia y grupo de amigos, relación que se va modificando o ampliando en el curso del desarrollo. El exosistema comprende entornos entre los cuales la persona no está como participante activa pero cuyos eventos influyen su desarrollo. Por ejemplo, el espacio laboral de los padres, los vínculos entre familia y comunidad, representando principalmente los ambientes sociales y organizaciones. El macrosistema está compuesto por los patrones culturales vigentes tales como creencias, ideologías, valores, sistemas políticos y económicos, organización de instituciones sociales y comunitarias en una particular cultura o subcultura. Este conjunto tiene mucho poder en las formas de relación que ocurren en los sistemas anteriores, ya que son internalizados de forma activa por el individuo, influenciando sus comportamientos. En general, se relaciona con los valores, las leyes y la cultura. Finalmente, el cuarto núcleo es el tiempo o cronosistema, que involucra eventos y rutinas de la persona a lo largo del ciclo vital, así como los acontecimientos históricos de determinada época (Shaffer, 2000). 


\section{La mirada de la resiliencia desde el modelo ecológico}

Ehrensaft y Tousignant (2003) utilizan el modelo ecológico de Bronfenbrenner $(1979,1987)$ para una mejor comprensión del niño resiliente en su entorno. En la ecología social del niño en riesgo y su descripción de los subsistemas incluyen un subsistema que se torna muy importante: el ontosistema, el cual estaría constituido por características internas. Teniendo en cuenta este punto de vista (Belsky, 1980, 1993), se sostiene que la persona incluida en el contexto posee una serie de capacidades, competencias y vulnerabilidades que forman parte del ontosistema (Morelato, 2009).

El ontosistema estaría constituido por los factores internos tanto biológicos (maduración del SNC, temperamento y genética) como psicológicos (calidad del apego, autoconcepto, capacidades cognitivas, afectivas y otros atributos) y además neuropsicológicos, los cuales son producto de la interacción entre los dos aspectos anteriores. Para explicar el proceso de interacción entre el individuo y su contexto se incluyen dos grandes dimensiones previamente mencionadas: la dimensión de riesgo y la dimensión de protección, como se observa en la figura 2 (Morelato, 2009). La interrelación entre ambas puede generar un mecanismo de protección que daría lugar a la resiliencia o, en caso contrario, un mecanismo de riesgo que lleva a la vulnerabilidad. En el marco de estas dos dimensiones ubicaremos al contexto, el cual corresponde al tercer núcleo.

Finalmente, el tiempo daría lugar al cuarto núcleo (cronosistema) en el que se destaca especialmente que el impacto de un factor de riesgo puede depender del momento de desarrollo evolutivo, lo cual se entiende mejor teniendo en cuenta que a edades más tempranas existe mayor predisposición a la vulnerabilidad (Gallardo Cruz, Trianes Torres \& Jiménez Hernández, 1998). Los factores de riesgo o los de protección abarcan todos los subsistemas, por lo tanto podrían ubicarse en cualquiera de ellos. La familia, los vínculos significativos y las características internas de estos miembros son parte del microsistema que incluyen su grado de estabilidad emocional, su historia y aspectos personales, entre otras cosas. 
El resto de los factores externos forman parte de otros subsistemas. El mesosistema, por ejemplo, involucra la calidad de las relaciones de la familia con la escuela, la comunidad, la familia extensa y el acceso a vías de contención social y educativa (escuela, hospital, municipio). Estas también pueden denominarse redes de apoyo secundarias. Asimismo, el exosistema estaría conformado por la relación entre las instituciones, si se trata de un ambiente urbano, rural, urbano marginal, pobre o de clase media, junto con el nivel socioeconómico y el tipo de población al que corresponde. Finalmente, el macrosistema está relacionado con las políticas sociales, la cultura del país y el momento histórico que transcurre como significativo para el desarrollo.

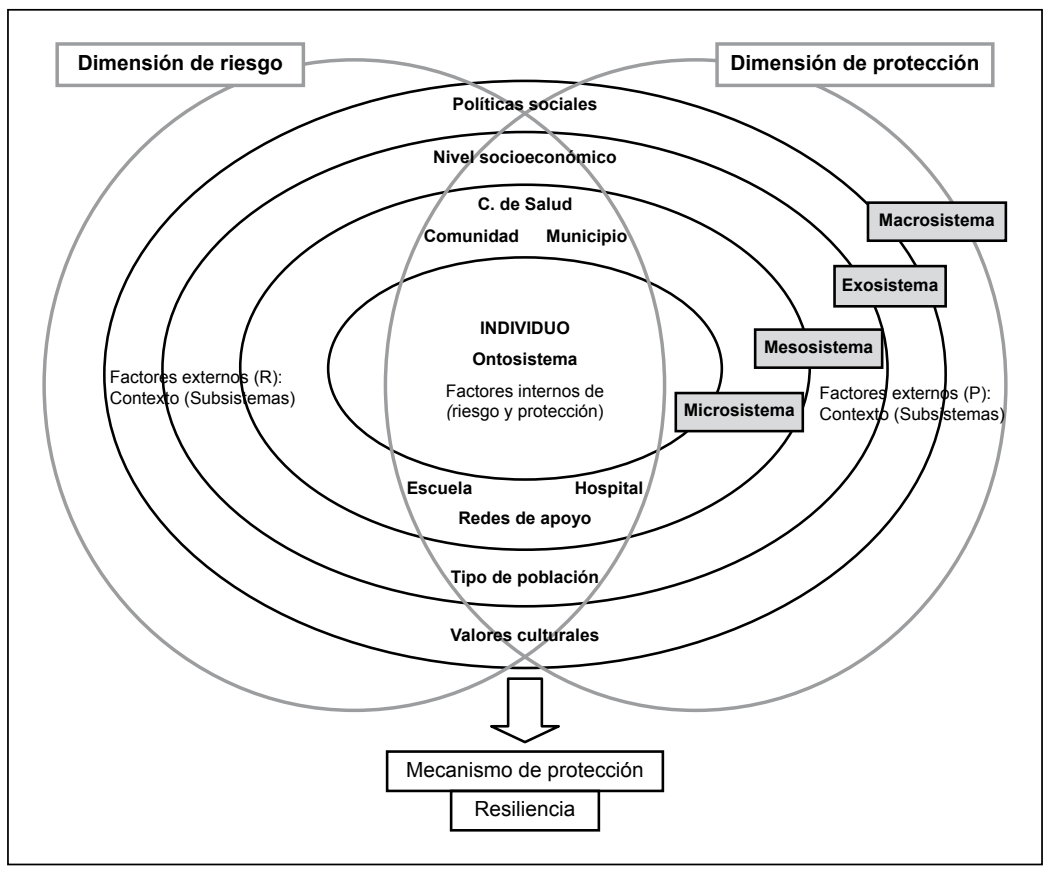

Figura 2. El proceso de resiliencia aplicado al modelo ecológico y adaptado por la autora (Morelato, 2009). 


\section{El modelo bioecológico aplicado a la comprensión de la resiliencia en el maltrato infantil}

Aunque existen numerosos modelos teóricos que buscan explicar el problema del maltrato infantil, actualmente el enfoque predominante es también el modelo ecológico (Trianes Torres \& Gallardo Cruz, 1997). Considerando que este modelo es utilizado para explicar el proceso de resiliencia, incorporaremos al mismo el análisis de los factores relacionados con la vulnerabilidad o la resiliencia en el ámbito del maltrato infantil. Es preciso aclarar que los elementos incluidos en cada uno de los subsistemas se relacionan entre sí de modo flexible y permeable, y su ubicación responde propósitos de claridad gráfica. Sin embargo, teniendo en cuenta el contexto específico del maltrato infantil, se puede decir que algunos factores son siempre de riesgo, como por ejemplo la presencia de un padre alcohólico o los indicadores de violencia y maltrato. Por otro lado, otros factores serán siempre protectores, por ejemplo, una persona que toma conciencia del problema y pide ayuda.

Es así que, considerando la relevancia de los factores que conforman la dimensión protectora, es posible pensar que la potenciación de un mejor funcionamiento y el fortalecimiento de esta dimensión podrían generar una dinámica compensatoria que amortiguaría el riesgo. Esto no significa que el maltrato no se instala en la historia del sujeto o no tiene efecto, sino que las redes y el sostén de los aspectos internos y externos darían continuidad al desarrollo a pesar de las circunstancias.

Para retomar el diagrama, partimos de la incorporación de los dos círculos concéntricos que representan las dimensiones de riesgo y de protección (ver figura 3). Cuando analizamos la dimensión de protección, en el ontosistema se destacan como elementos protectores las competencias personales tales como un nivel intelectual normal (Kotliarenco et al., 1997), las habilidades sociales en sentido general y especialmente las habilidades de solución de problemas interpersonales (Nears, 2004), la creatividad (Cyrulnik, 2003b; Fuentes \& Torbay, 
2004) y el autoconcepto (Morelato, 2009), asociado al haber internalizado alguna buena figura de apego (Amar Amar, Kotliarenco \& Abello Ramos, 2003; Cicchetti et al., 1993; Cicchetti \& Rogosch, 1997).

En contraste, desde la dimensión de riesg, podríamos pensar que existen aspectos propios o características internas que funcionarían como eventualidades o dificultades para el desarrollo. Estos aspectos pueden ser la presencia de problemas de conducta (agresión, inhibición) o déficit en habilidades de interacción social, trastornos psiquiátricos (depresiones, psicosis), problemas intelectuales, baja autoestima, tendencia al locus de control externo, falta de capacidad reflexiva, de empatía y haber tenido una historia de apego disfuncional, entre otras problemáticas. Los factores personales no son determinantes pero inciden negativamente en la interacción con el contexto, generando mayor probabilidad de desarrollar vulnerabilidad. Es por ello que consideramos pertinente estimular las competencias protectoras mencionadas tanto en forma individual o grupal, a fin de fortalecer las posibilidades personales de enfrentar el riesgo (modelo de desafío), compensarlo (modelo compensatorio) o atenuarlo (modelo de adaptación).

En sintonía con lo anterior y a nivel del microsistema, desde la perspectiva de la protección pueden situarse las características de los miembros de la familia nuclear. Entre las más importantes están las funciones de apoyo de aquellos miembros, si los hay, que presenten una buena señal de alarma, es decir, que tomen conciencia de la desprotección infantil. Por ello, se sostiene que si existen del lado del riesgo señales de violencia o maltrato, es posible potenciar la resiliencia si nos enfocamos en aquellas personas que perciben que existe algún problema en la familia, lo denuncian o buscan ayuda. De este modo, podemos detectar recursos para cuidar al nińo concretamente. Por ejemplo, si nos encontramos con una familia donde prima el maltrato físico por parte de un cuidador podemos observar si uno de los miembros de la pareja ha denunciado y ha podido sostener la exclusión de la persona violenta, o si hay un abuelo/a, tío/a o padrino/madrina que está dispuesto a resguardar y hacerse cargo del niño. Esto facilita los mecanismos protectores en la medida que se sustenta el apoyo, el cual interactúa con el 
riesgo sufrido sumado a las características personales del niño y de los cuidadores.

Además, el afecto, las habilidades intelectuales e interpersonales, y la capacidad de reflexión y empatía de aquellos integrantes adultos de la familia que están en contacto con los niños, posibilitan la flexibilidad, la comunicación y la cohesión familiar mencionadas por Walsh (1996) como aspectos de resiliencia. Por otra parte, forman parte del microsistema el grupo de pares, el sistema escolar y la red familiar extensa. Las redes formales (escuela, iglesia, ámbito de salud) e informales (vecinos, miembros de la comunidad, amigos) resultan altamente protectoras porque son grupos humanos que brindan contención, lo cual va en sentido opuesto al aislamiento, señalado como una de las causas del maltrato (Moreno Manso, 2005).

En esta línea y en la dimensión de riesgo del microsistema, hallamos los indicadores específicos e inespecíficos de maltrato familiar. Sin embargo, también se incluyen aspectos menos severos pero importantes tales como la incongruencia o inconsistencia en la puesta de límites, las dificultades en la comunicación familiar, la presencia de historia de maltrato en los progenitores y el hacinamiento, entre otros, lo cual puede ser la antesala de episodios más graves. Siguiendo con esta dimensión, en el mesosistema, pueden darse fallas de comunicación entre la familia y las instituciones tales como la escuela y la comunidad. Por ejemplo, un niño que no asista a la escuela o se fugue sin que la familia de cuenta de la razón ni colabore con la inserción. Asimismo, en muchas oportunidades el grupo de pares también se torna problemático cuando ofrece una identidad de pertenencia relacionada con el conflicto. Otro aspecto es la inseguridad y la violencia en la comunidad, la falta de información respecto al cuidado de la salud y del propio cuerpo, lo cual puede llevar muy habitualmente a un inicio sexual muy precoz y embarazos no deseados.

No obstante, si en la dimensión de protección del mesosistema enfatizamos la función de los miembros de la familia extensa (abuelos, tíos, padrinos) y las buenas relaciones con la comunidad, podemos implementar programas de ayuda. Incluso, la participación de la 
familia en una comunidad religiosa resulta importante para el desarrollo de procesos de resiliencia, ya que ofrece sostén y asistencia moral o espiritual como elemento amortiguador (Kotliarenco et al., 1997). El club o la unión vecinal también son beneficiosos cuando organizan actividades de modo tal que fomentan el sentimiento de pertenencia, fortalecen el rol del grupo de pares y ponen en juego habilidades de interacción social. Asimismo, es valiosa la colaboración entre familia y comunidad a través de los docentes en la escuela y su preocupación por el bienestar infantil. Por otra parte, el seguimiento que realizan los equipos de atención primaria de la salud en los controles de rutina y el rol de los equipos especializados en la temática de violencia son esenciales en la protección, ya sea para buscar un adulto que ejerza una función materna adecuada o para incorporar alguien de la familia extensa o en su defecto a familias cuidadoras incorporadas al sistema judicial. Sin embargo, este elemento protector por sí mismo es insuficiente si no se fortalecen además habilidades internas en los niños y cuidadores que faciliten procesos de resiliencia.

Siguiendo este esquema, podemos pensar que en el exosistema la dimensión de riesgo se compone por las dificultades que poseen los adultos para tener oportunidad de acceso al empleo y a una adecuada inserción social, sumado a los problemas económicos y a la pobreza. Indirectamente, las fallas en la implementación de políticas públicas de protección a la infancia, de salud y de educación viables se manifiestan en la falta de coordinación entre la policía, los organismos de salud, escolares y judiciales. Esta desintegración se vuelve una revictimización para el nińo y para la familia cuando ocurren situaciones tales como repitencia de revisaciones médicas invasivas, evaluaciones psicológicas o psiquiátricas, exposición a procesos que pueden ser aún más violentos que el maltrato sufrido, traslados reiterados, no realización de procesos de exclusión a tiempo, demoras excesivas en los turnos de salud o judiciales y derivaciones consecutivas sin sentido. En este subsistema, si bien ni el niño ni su familia tienen participación activa, sufren sus consecuencias. 
Para concluir con la dimensión de riesgo, se sostiene que a nivel del macrosistema las creencias rígidas y descalificadoras respecto de los roles de género y el lugar del menor en la sociedad, la naturalización de la violencia, la idea de que el castigo corporal es un modo de educación que "endereza", forman parte de los conceptos subyacentes tanto culturales como subculturales que están en la base del maltrato y la violencia, y se traducen en falta de leyes que apoyen los derechos de los nińos. Por ello, desde el punto de vista de la dimensión protectora, es necesario implementar buena comunicación entre las redes formales e informales, es decir coordinaciones entre la escuela, el hospital, y los ámbitos municipales, judiciales y comunitarios, a fin de incidir en los procesos que den continuidad al desarrollo infantil. En consecuencia, no deben descuidarse recursos tales como planes de emergencia social, organizaciones de la sociedad civil, comedores infantiles, programas de capacitación laboral y proyectos subsidiados por organismos nacionales $\mathrm{o}$ internacionales, entre otros.

El funcionamiento de los organismos gubernamentales tales como policía, juzgados, instituciones de salud, escolares y comunitarias es prioritario a fin de mantener el interés superior del nińo y el apoyo a las víctimas. La denuncia debería ser la primer visagra del sistema de protección ejecutando una función de límite en el proceso de violencia. A su vez, el exosistema se torna protector cuando la red social está nutrida por la capacitación de profesionales de la salud, agentes comunitarios, docentes, funcionarios públicos y personas formadas en los temas de maltrato y violencia. La información y el compromiso los incentiva a tomar parte del problema desde su lugar de ciudadanos ejerciendo un rol participativo y activo. Por otra parte, la protección se incrementa en gran medida en esta dimensión cuando se articulan acciones por medio de políticas viables que apoyan la economía, el trabajo, la salud y la educación. Estas acciones forman parte del macrosistema.

Además, cuando circula la creencia de que los castigos físicos o psicológicos no son modos de educar y cuando culturalmente se instala la idea de su desnaturalización, podemos pensar que estamos avanzando hacia la protección de la infancia maltratada. Esto también subyace 
cuando se crean espacios de discusión, información, prevención, asistencia y programas de apoyo psicosocial destinados a niños, padres, docentes y profesionales. En estos espacios pertenecientes al meso y exosistema, se ponen en discusión valores tales como la situación de la infancia, el trabajo infantil o el rol de la mujer desde una postura adaptada a los momentos históricos actuales (cronosistema), entre otros valores humanos. Podemos sintetizar este último punto diciendo que, para la dimensión protectora del macrosistema, resulta importante la revalorización del niño como sujeto de derechos y que esto se manifieste en el ámbito de la legislación del país y la provincia.

El presente trabajo se propuso realizar un breve recorrido sobre los principales postulados acerca de la resiliencia, incorporando un análisis de los factores de riesgo y de los factores de protección en el ámbito del maltrato infantil y en el marco del modelo ecológico. El integrar conceptos vinculados a la resiliencia y al maltrato infantil a través de este modelo intenta acercarnos a la comprensión de este constructo como proceso dinámico. En el ámbito del maltrato infantil, la resiliencia dependerá fundamentalmente de dos cosas: por un lado de los propios recursos, es decir de los factores internos asociados al ontosistema y, por otra parte, dependerá de los factores externos asociados al micro, meso, exo y macrosistema.

Los factores involucrados podrán pertenecer tanto a la dimensión de riesgo como a la dimensión de protección, e interactuarán recíprocamente dando lugar a un mecanismo de resiliencia o de vulnerabilidad. En el maltrato infantil, tal como lo plantean Cicchetti y Rogosch (2001), la presencia de indicadores de maltrato de diversos niveles de cronicidad y severidad estarían formando parte del microsistema y serían uno de los aspectos del alto riesgo para el funcionamiento cognitivo, biológico y social del nińo, es decir, para su ontosistema. Es por ello que se sostiene que al potenciar factores protectores de los cuatro subsistemas se podrían facilitar mecanismos de protección a modo de modelos compensatorios, de desafío o de adaptación (Werner, 1993), es decir, potenciar la generación de procesos de resiliencia. 


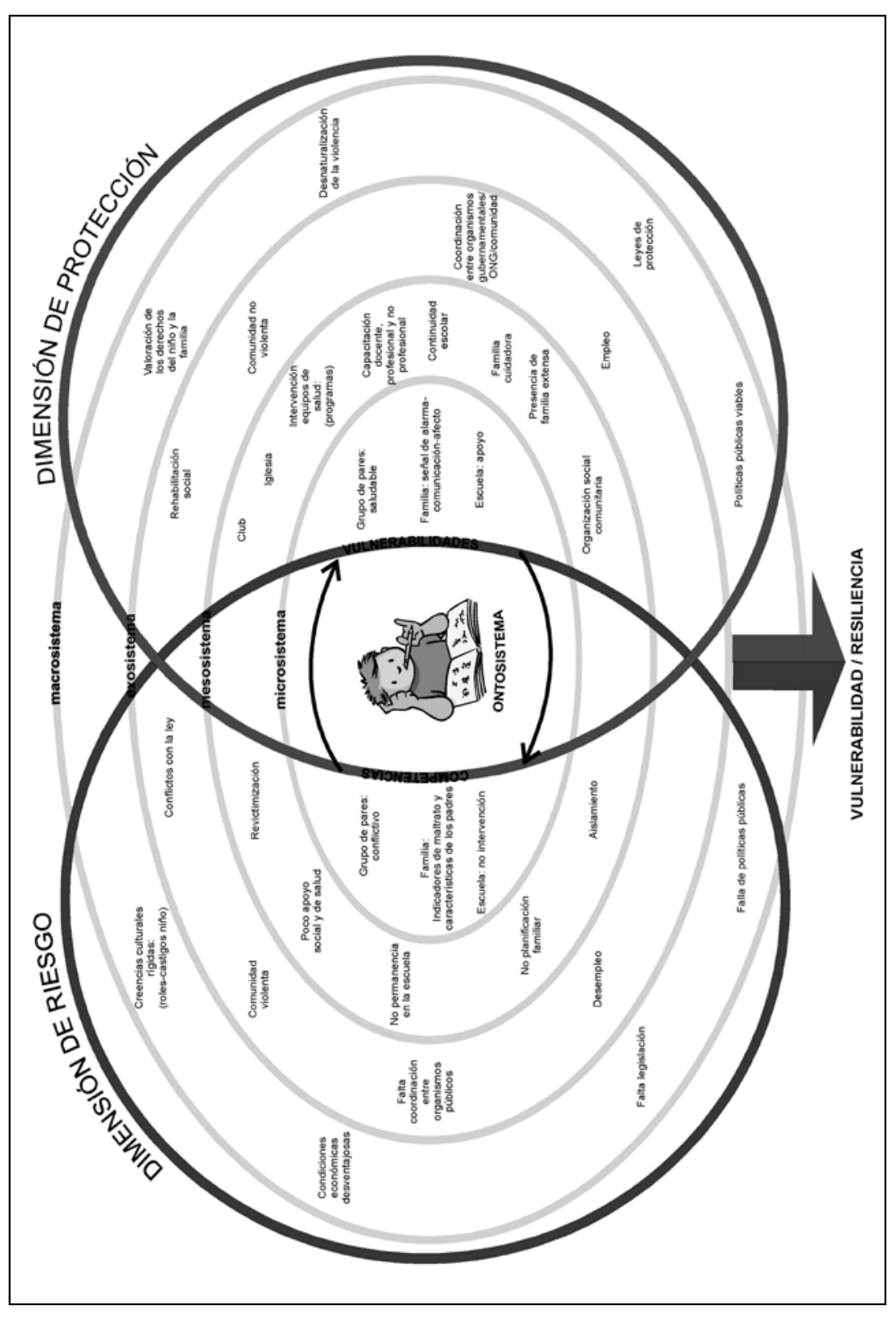

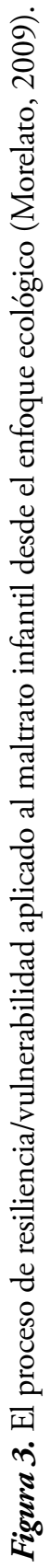


En la línea de lo expuesto, nuestro lugar de agentes de salud mental señala una alternativa válida para el desarrollo de los procesos de resiliencia si nos incorporamos como parte del contexto infantil. Desde el microsistema, por ejemplo, sería esencial el seguimiento cercano de la familia y los niños en riesgo, tanto desde los servicios de protección familiar como desde la escuela. En estos ámbitos sería importante implementar programas que fortalezcan recursos y competencias en los niños (e. g. autoestima, habilidades de solución de problemas, habilidades cognitivas y creatividad, entre otros aspectos). Además podemos potenciar mecanismos protectores si desde el punto de vista del mesosistema colaboramos con la vinculación de la familia y los niños tanto con las docentes de la escuela, como con los líderes comunitarios y demás actores sociales que pueden generar redes de apoyo.

Estas conexiones y redes fortalecen aspectos protectores del exosistema en la medida que se incentivan comunicaciones claras entre las instituciones sanitarias, escolares y sociales, a fin de lograr seguimientos que tengan coherencia y fluidez. También sería importante propiciar espacios de discusión en el ámbito de las políticas públicas, lo cual tendría relación con el macrosistema. De este modo, tal como lo plantea Grotberg (1997), al incentivar en los nińos sus fortalezas y recursos en sí mismos y a su alrededor se potencian factores protectores, lo cual podrá llevarlos a actuar y a superar las dificultades, y se atenuaría el impacto del riesgo.

Finalmente es preciso señalar que, aunque las posturas críticas expresan que la teoría de la resiliencia no agrega nada a las teorías del desarrollo ya que se trataría simplemente de un ajuste positivo, tal como plantean Luthar et al. (2000) la resiliencia es un constructo distintivo de otras teorías del desarrollo porque proporciona un marco conceptual que contrasta con las teorías clásicas. En ese marco y a través del logro de un ajuste positivo en la fase de adversidad, la teoría de la resiliencia presenta una nueva mirada sobre las trayectorias del desarrollo que difieren de lo esperable, es decir de lo normativo, pero que aún así son comunes. En esta línea, comprendiendo los factores protectores y los factores de riesgo del maltrato infantil desde el punto de vista del 
modelo ecológico, podemos acercarnos a procesos que contribuyen a ese ajuste y es posible ampliar la comprensión de los procesos de desarrollo humano cuando los entornos no son adecuados.

Por lo tanto, y sin subestimar el alto riesgo que conlleva la problemática del maltrato, es necesario incorporar nuevos modos de comprensión del desarrollo infantil donde se considere que luego de resguardar al niño de la situación familiar de violencia, aspecto prioritario y fundamental, es necesario fortalecer factores protectores en el niño y en la comunidad desde los programas que estimulen la resiliencia. Asimismo, resulta importante continuar la investigación en esta línea por medio de trabajos empíricos que iluminen el camino de las intervenciones en resiliencia y maltrato infantil, y precisen nuevas modalidades de abordaje de la problemática.

\section{Referencias}

Amar Amar, J. J., Kotliarenco, M. \& Abello Llanos, R. (2003). Factores psicosociales asociados con la resiliencia en niños colombianos víctimas de violencia intrafamiliar. Investigación y Desarrollo, 11(1), 162-197.

Baldwin, A. L., Baldwin, C. \& Cole, R. E. (1992). Stress-resistant families and stress-resistant children. En J. Rolf, A. Masten, D. Cicchetti, K. Nuechterlein \& S. Weintraub (Eds.), Risk and protective factors in the development of psychopathology. Cambridge, Reino Unido: Cambridge University Press.

Belsky, J. (1980). Child maltreatment: An ecological integration. American Psychologist, 35, 86-96.

Belsky, J. (1993). Etiology of child maltreatment: A developmental ecological analysis. Psychological Bulletin, 3, 83-96.

Bronfenbrenner, U. (1979). The ecology of human development: Experiments by nature and design. En D. Shaffer, Psicología del desarrollo. Infancia y adolescencia. (5a. ed.) México: Thompson. 
Bronfenbrenner, U. (1987). La ecología el desarrollo humano: experimentos en entornos naturales y diseñados. Barcelona: Paidós.

Casol, L. \& De Antoni, C. (2006). Família e abrigo como rede de apoio social e afetiva. En D. Dalbosco Dell'Aglio, S. Koller \& M. A. Matter Yunes (Eds.), Resiliencia e psicología positiva: Interfaces do risco à proteçao. Sao Paulo: Casa do Psicólogo.

Cicchetti, D. (2001). The impact of social experience on neurobiological systems: Illustration from a constructivist view of child maltreatment. Cognitive Development, 17(3-4), 1407-1428.

Cicchetti, D. \& Lynch, M. (1993). Toward an ecological/transactional model of community violence and child maltreatment: Consequences for children's development. Psychiatry, 56, 96-117.

Cicchetti, D. \& Rogosch, F. (1997). The role of self-organization in the promotion of resilience in maltreated children. Development and Psychopathology, 9, 797-815.

Cicchetti, D., Rogosch, F., Lynch, M. \& Holt, K. (1993). Resilience in maltreated children: Processes leading to adaptive outcome. Development and Psychopathology, 5, 629-647.

Cicchetti, D. \& Rogosch, F. (2001). Diverse patterns of neuroendocrine activity in maltreated children. Development and Psychopatho$\log y$, 13, 677-693.

Cyrulnik, B. (2003a). El murmullo de los fantasmas. Madrid: Gedisa.

Cyrulnik, B. (2003b). Los patitos feos. La resiliencia: una infancia infeliz no determina la vida. Madrid: Gedisa.

Flores, E., Cicchetti, D. \& Rogosch, F. (2005). Predictors of resilience in maltreated and non-maltreated Latino children. Developmental Psychology, 41(2), 338-351.

Fuentes Ramos, C. \& Torbay Betancor, A. (2004). Desarrollar la creatividad desde los contextos educativos: un marco de reflexión sobre la mejora socio-personal. Revista Electrónica Iberoamericana sobre Calidad, Eficacia y Cambio en Educación, 2(1). Recuperado de http: // www.ice.deusto.es /rinace/reice/vol2n1/ Fuentes.pdf 
Gallardo Cruz, J. A., Trianes Torres, M. \& Jiménez, M. (1998). El maltrato fisico hacia la infancia. Sus consecuencias socioafectivas. Málaga: Universidad de Málaga.

Grotberg, E. (1997). La resiliencia en acción. Seminario Internacional sobre Aplicación del Concepto de Resiliencia en Proyectos Sociales, Fundación Van Leer/Universidad Nacional de Lanús, Argentina.

Kotliarenco, M. A., Cáceres, I. \& Fontecilla, M. (1997). Estado del arte en resiliencia. Santiago de Chile: Organización Panamericana de la Salud/Fundación Kellogg/Agencia Sueca de Cooperación Internacional para el Desarrollo.

Lessinger Borges, J., Haag Kristensen, C. \& Dalbosco Dell'Aglio, D. (2006). Neuroplasticidade e resiliencia em crianças e adolescentes vítimas de maus-tratos. En D. Dalbosco Dell'Aglio, S. Koller \& M. A. Matter Yunes (Eds.), Resiliencia e psicología positiva: Interfaces do risco à proteçao. Sao Paulo: Casa do Psicólogo.

Luthar, S. S. (1991). Vulnerability and resilience: A study of high risk in adolescents. Child Development, 62(3), 600-616.

Luthar, S. S. (1993). Annotation: Methodological and conceptual issues in research on childhood resilience. Journal of Child Psychology and Psychiatry, 34(4), 441-453.

Luthar, S. S., Cicchetti, D. \& Becker, B. (2000). The construct of resilience: A critical evaluation and guidelines for future work. Child Development, 71(3), 543-562.

Melillo, A. \& Suárez Ojeda, N. (2001). Resiliencia. Descubriendo las propias fortalezas. Buenos Aires: Paidós.

Melillo, A., Suárez Ojeda, N. \& Rodríguez, D. (2004). Resiliencia y subjetividad. Buenos Aires: Paidós.

Morelato, G. (2005). Perspectivas actuales de los procesos que sustentan la resiliencia infantil. Investigaciones en Psicología, 10(2), 61-82.

Morelato, G. (2009). Evaluación de la resiliencia en niños víctimas de maltrato familiar (Tesis doctoral inédita). Universidad Nacional de San Luis, Argentina. 
Moreno Manso, J. (2005). Maltrato infantil: un estudio sobre la familia, la red de apoyo social y las relaciones de pareja. Recuperado de http:// www.psiquiatria.com/articulos/trastornos infantiles/22714.

Nears, K. (2004). Resilience in maltreated children (Tesis). Recuperado de http://www.lib.ncsu.edu

Rutter, M. (1990). Psychosocial resilience and protective mechanisms. En J. Rolf, A. Masten, D. Cicchetti, K. Nucchterlein \& S. Weintraub (Eds.), Risk and protective factors in the development of psychopathology. Nueva York: Cambridge University Press.

Rutter, M. (1995). Resiliencia: algunas consideraciones sobre su concepto. Contemporary Pediatrics (Edición Argentina), 3(3), 25-38.

Shaffer, D. (2000). Psicología del desarrollo. Infancia y adolescencia (5a. ed.) México: Thomson.

Trianes Torres, M. V. \& Gallardo Cruz, J. A. (1997). Influencia del sexo y la edad en las repercusiones psicológicas de los niños maltratados físicamente. Psicothema, 9(3), 473-485.

Walsh, F. (1996). The concept of family resilience: Crisis and challenge. Family Process, 35, 261-281.

Werner, E. \& Smith, R. S. (1982). Vulnerable but invincible: A longitudinal study of resilient children and youth. Nueva York: McGraw Hill.

Werner, E. (1993). Protective factors and individual resilience. En S. Meisels \& J. Shonkof (Eds.), Handbook of early childhood intervention. Nueva York: Cambridge University Press.

Recibido: 14 de julio, 2010 Aceptado: 25 de enero, 2011 